\title{
A tAXONOMIA II PROPOSTA PELA NORTH AMERICAN NURSING DIAGNOSIS ASSOCIATION (NANDA)
}

Diná de Almeida Lopes Monteiro da $\mathrm{Cruz}^{2}$

Braga CG, Cruz DALM. A Taxonomia II proposta pela North American Nursing Diagnosis Association (NANDA). Rev Latino-am Enfermagem 2003 março-abril; 11(2):240-4.

Este artigo tem a finalidade de divulgar o desenvolvimento e as características da estrutura multiaxial da Taxonomia II, da NANDA. Essa estrutura oferece maior flexibilidade para os enunciados diagnósticos e é mais adequada às práticas de enfermagem contemporâneas que a Taxonomia $I$.

DESCRITORES: classificação, diagnóstico de enfermagem

\section{THE TAXONOMY II PROPOSED BY THE NORTH AMERICAN NURSING DIAGNOSIS ASSOCIATION (NANDA)}

This article aims to disseminating the development and characteristics of NANDA's Taxonomy II multiaxial structure. This structure offers greater flexibility to diagnoses statements and is more adequate to the contemporaneous nursing practices than the Taxonomy I.

DESCRIPTORS: classification, nursing diagnosis

\section{LA TAXONOMÍA II PROPUESTA POR LA “NORTH AMERICAN NURSING DIAGNOSIS ASSOCIATION" (NANDA)}

Este artículo tiene la finalidad de divulgar el desarrollo y la estructura multiaxial de la Taxonomía II de la NANDA. La Taxonomía II ofrece mayor flexibilidad a los enunciados diagnósticos y es más adecuada a las prácticas de enfermería contemporánea que la Taxonomía I.

DESCRIPTORES: clasificación, diagnóstico de enfermería

\footnotetext{
${ }^{1}$ Enfermeira, Mestre em Fundamentos de Enfermagem, Doutoranda em Enfermagem, e-mail: cristianegbraga@uol.com.br; ${ }^{2}$ Enfermeira, Professor Livre-Docente, e-mail: mtmllf@usp.br. Escola de Enfermagem da Universidade de São Paulo
} 
INTRODUÇÃO

$A$ responsabilidade do cuidar em enfermagem exige que as decisões sobre as intervenções propostas sejam fundamentadas na avaliação do estado de saúde do indivíduo. Essa avaliação requer que se adote o diagnóstico de enfermagem como referência.

Como instrumento de trabalho da enfermeira, o diagnóstico é "uma forma de expressar as necessidades de cuidados que identificamos naqueles de quem cuidamos. Por exemplo, ao avaliarmos um doente internado e concluirmos que ele tem alto risco para lesão de pele, essa afirmação - alto risco para lesão de pele, ou alto risco para integridade da pele prejudicada - nada mais é do que a expressão de uma situação do doente que, em nosso julgamento, necessita de intervenção de enfermagem. Se é uma situação que necessita de intervenção de enfermagem, é uma necessidade de cuidado. Com isso, podemos, então, afirmar que diagnósticos de enfermagem expressam necessidades de cuidados"(1)

Os diagnósticos de enfermagem são os focos clínicos da ciência de enfermagem ${ }^{(2-3)}$, e a atividade diagnóstica aproxima as enfermeiras de seus clientes, possibilitando-lhes conhecer melhor suas respostas físicas e emocionais $^{(4)}$.

A NANDA, contribuindo de forma significativa para o desenvolvimento e refinamento dos diagnósticos de enfermagem, tem desenvolvido um sistema conceitual para classificar os diagnósticos em uma taxonomia ${ }^{(5)}$. Em 1989, publicou a Taxonomia I e, com base em diversas avaliações, publicou, em 2001, a Taxonomia II cuja estrutura foi aceita em sua conferência bienal em $2000^{(6)}$.

Este artigo tem a finalidade de divulgar o desenvolvimento e as características da estrutura multiaxial da Taxonomia II. Para isso, utilizou-se como fonte principal o livro NANDA - Nursing Diagnoses: definitions and classification - 2001 e 2002.

\section{DESENVOLVIMENTO DA TAXONOMIA II}

O principal trabalho da NANDA é direcionar a padronização de linguagem dos diagnósticos. Padronizar a linguagem é estabelecer um acordo sobre regras para utilização de determinados termos ${ }^{(7)}$.

Até 2000, a NANDA classificava os diagnósticos de enfermagem numa estrutura designada Taxonomia I, que era constituída por nove categorias chamadas de Padrões de Respostas Humanas.

A classificação em Padrões de Respostas Humanas vinha sendo bastante criticada. Em 1994, o Comitê de Taxonomia - parte da NANDA - enfrentando sérias dificuldades para classificar novos diagnósticos que eram aceitos, reconheceu que era viável uma nova estrutura para a classificação. Decidiu, então, verificar se surgiriam conjuntos "naturais" de diagnósticos utilizando o método "Q-sort" naturalístico*, cuja primeira rodada concluiu-se na conferência da NANDA de 1994.

A segunda rodada foi concluída mais tarde, e os resultados foram apresentados na conferência de 1996. A estratégia permitiu identificar 21 categorias para classificarem-se os diagnósticos, e esse era um número muito grande que prejudicaria a sua utilidade como estrutura de classificação(1).

Em 1998, o Comitê de Taxonomia organizou e enviou à diretoria da NANDA quatro resultados de "Q-sort", usando diferentes estruturas: a primeira era o resultado do "naturalístico" realizado entre 1994 e 1996; a segunda usava a estrutura proposta por Jenny ${ }^{(8)}$; a terceira usava a estrutura da Nursing Outcomes Classification (NOC) ${ }^{(9-10)}$, e a quarta tinha como base os Padrões Funcionais de Saúde ${ }^{(11-12)}$. Considerou-se que nenhuma dessas estruturas era completamente satisfatória, mas que a dos Padrões Funcionais de Saúde ${ }^{(11-12)}$ era a melhor delas. Assim, com a permissão da autora, a NANDA modificou a estrutura dos Padrões Funcionais de Saúde ${ }^{(11-12)}$ e criou uma quinta alternativa que foi apresentada a seus membros em 1998. Nessa conferência, os membros foram convidados a distribuir os diagnósticos já aceitos entre os domínios que essa quinta estrutura previa. Dessa etapa, resultaram 40 conjuntos de respostas possíveis de serem analisados. Durante a coleta desses dados, o Comitê de Taxonomia anotou os comentários, questões e dificuldades expressas pelos participantes, bem como as sugestões feitas para melhorar a estrutura em estudo. Com essas notas e os conjuntos de respostas, foram feitas modificações adicionais à estrutura: divisão de um domínio em dois, criação de domínio que não existia e renomeação

\footnotetext{
* Com esse método, solicitou-se a várias pessoas que agrupassem os diagnósticos existentes livremente, isto é, sem considerar a classificação então em uso pela NANDA, com a finalidade de identificar formas alternativas de organizar a classificação
} 
de domínios para que melhor espelhassem os diagnósticos neles contidos. Na visão da NANDA, publicada em 2001, a estrutura taxonômica final é pouco parecida com a proposta original de Gordon, mas reduziu os erros de classificação e redundâncias a valores muito próximos de zero, o que é uma condição altamente desejável para uma estrutura taxonômica ${ }^{(1)}$.

\section{ESTRUTURA DA TAXONOMIA II}

No final do processo de revisão, a estrutura aceita para substituir a classificação pautada nos Padrões de Respostas Humanas (Taxonomia I) continha domínios e classes. Assim, na Taxonomia II, cada domínio é composto por classes e cada classe é composta por conceitos diagnósticos.

Foram também desenvolvidas definições para cada domínio e para cada classe da estrutura. A definição de cada diagnóstico foi comparada às definições da classe e do domínio onde eles estavam localizados e isso fundamentou a revisão e modificações nas localizações dos diagnósticos com a finalidade de assegurar a máxima consistência entre diagnósticos, classes e domínios. A Taxonomia II, publicada em 2001, tem 13 domínios, 106 classes, 155 diagnósticos e sete eixos ${ }^{(1)}$.

A Tabela 1 mostra os domínios e classes da Taxonomia II ${ }^{(6)}$. Nela apresentamos, também, os termos no original em inglês, visto que esta não é uma tradução oficial.

Tabela 1 - Taxonomia II da NANDA: domínios e classes

\begin{tabular}{|c|c|}
\hline Domínios & Classes \\
\hline $\begin{array}{l}\text { Prom oção da Saúde } \\
\text { (Health Promotion) }\end{array}$ & $\begin{array}{l}\text { 1. Consciência de Saúde (Health Awareness) } \\
\text { 2. Controle da Saúde (Health Management) }\end{array}$ \\
\hline 2. Nutrição (Nutrition) & $\begin{array}{l}\text { 1. Ingestão (Ingestion) } \\
\text { 2. Digestão (Digestion) } \\
\text { 3. Absorção (Absortion) } \\
\text { 4. Metabolismo (Metabolism) } \\
\text { 5. Hidratação (Hydration) }\end{array}$ \\
\hline 3. Eliminação (Elimination) & $\begin{array}{l}\text { 1. Sistema Urinário (Urinary System) } \\
\text { 2. Sistema Gastrintestinal (Gastrointestinal System) } \\
\text { 3. Sistema Tegumentar (Integumentary System) } \\
\text { 4. Sistema Pulmonar (Pulmonary System) }\end{array}$ \\
\hline 4. Atividade / Repouso (Activity / Rest) & $\begin{array}{l}\text { 1. Sono / Repouso (Sleep / Rest) } \\
\text { 2. Atividade / Exercício (Activity / Exercise) } \\
\text { 3. Equilíbrio de Energia (Energy Balance) } \\
\text { 4. Respostas Cardiovasculares / Pulmonares } \\
\text { (Cardiovascular / Pulmonar Responses) }\end{array}$ \\
\hline $\begin{array}{l}\text { 5. Percepção / Cognição } \\
\text { (Perception / Cognition) }\end{array}$ & $\begin{array}{l}\text { 1. Atenção (Attention) } \\
\text { 2. Orientação (Orientation) } \\
\text { 3. Sensação/Percepção (Sensation / Perception) } \\
\text { 4. Cognção (Cognition) } \\
\text { 5. Comunicação (Communication) }\end{array}$ \\
\hline 6. Autopercepção (Self-Perception) & $\begin{array}{l}\text { 1. Autoconceito (Self-Concept) } \\
\text { 2. Auto-estima (Self-Esteem) } \\
\text { 3. Imagem Corporal (Body Image) }\end{array}$ \\
\hline $\begin{array}{l}\text { 7. Relacionamentos de Papel } \\
\text { (Role Relationships) }\end{array}$ & $\begin{array}{l}\text { 1. Papéis de Cuidador (Caregiving Roles) } \\
\text { 2. Relacões Familiares (Family Relationships) } \\
\text { 3. Desempenho de Papel (Role Performance) }\end{array}$ \\
\hline 8. Sexualidade (Sexuality) & $\begin{array}{l}\text { 1. Identidade Sexual (Sexual Identity) } \\
\text { 2. Função Sexual (Sexual Function) } \\
\text { 3. Reprodução (Reproduction) }\end{array}$ \\
\hline $\begin{array}{l}\text { 9. Enfrentam ento / Tolerância ao estresse } \\
\text { (Enfrentamento / Stress Tolerance) }\end{array}$ & $\begin{array}{l}\text { 1. Resposta Pós Trauma (Post-Trauma Response) } \\
\text { 2. Respostas de Enfrentamento (Coping Responses) } \\
\text { 3. Estresse Neuro-comportamental } \\
\text { (Neurobehavioral Stress) }\end{array}$ \\
\hline 10. Princípios de Vida (Life Principles) & $\begin{array}{l}\text { 1. Valores (Values) } \\
\text { 2. Crenças (Beliefs) } \\
\text { 3. Congruência entre Valor / Crença / Ação } \\
\text { (Value / Belief / Action Congruence). }\end{array}$ \\
\hline $\begin{array}{l}\text { 11. Segurança / Proteção } \\
\text { (Safety / Protection) }\end{array}$ & $\begin{array}{l}\text { 1. Infecção (Infection) } \\
\text { 2. Lesão Física (Physical Injury) } \\
\text { 3. Violência (Violence) } \\
\text { 4. Perigos Ambientais (Environmental Hazards) } \\
\text { 5. Processos Defensivos (Defensive Processes) } \\
\text { 6. Termorregulação (Thermoregulation) }\end{array}$ \\
\hline 12. Conforto (Comfort) & $\begin{array}{l}\text { 1. Conforto Físico (Physical Com fort) } \\
\text { 2. Conforto Ambiental (Environmental Comfort) } \\
\text { 3. Conforto Social (Social Com fort) }\end{array}$ \\
\hline $\begin{array}{l}\text { 13. Crescimento / Desenvolvimento } \\
\text { (Growth / Development) }\end{array}$ & $\begin{array}{l}\text { 1. Crescimento (Growth) } \\
\text { 2. Desenvolvimento (Development) }\end{array}$ \\
\hline
\end{tabular}


Na Tabela 1, podem-se observar as classes compreendidas pelos domínios. Cada classe congrega um ou mais conceitos diagnósticos (diagnostic concepts), e, sob cada conceito diagnóstico, a NANDA dispõe os diagnósticos já aceitos para testes clínicos. A Tabela 2 mostra um exemplo dessa estrutura.

Tabela 2 - Exemplo de diagnósticos aceitos na estrutura de Taxonomia II

\begin{tabular}{ll}
\hline \multicolumn{1}{c}{ Nível } & \multicolumn{1}{c}{ Conteúdo } \\
\hline Domínio 11 (Domain 11) & Segurança e proteção (Safety / Protection) \\
Classe 2 (Class 2) & Lesão Física (Physical Injury) \\
$\begin{array}{l}\text { Conceito Diagnóstico (Diagnostic } \\
\text { Concept) }\end{array}$ & Integridade da Pele (Skin Integrity) \\
$\begin{array}{l}\text { Diagnósticos aprovados } \\
\text { (Approved Diagnoses) }\end{array}$ & $\begin{array}{l}\text { Integridade da pele prejudicada } \\
\text { (Impaired skin integrity) }\end{array}$ \\
& $\begin{array}{l}\text { Risco para integridade da pele prejudicada } \\
\text { (Risk for imparied skin integrity). }\end{array}$ \\
\hline
\end{tabular}

Por exemplo: "integridade da pele" (skin integrity), na Taxonomia II, é um conceito diagnóstico. Sob o conceito diagnóstico "integridade da pele", estão as afirmações diagnósticas: "integridade da pele prejudicada" e "risco para integridade da pele prejudicada". Observe-se que, no conceito diagnóstico (integridade da pele), não há qualquer indicação que nos permita qualificar a "integridade da pele", isto é, qual é a situação de funcionalidade da "integridade da pele". Por outro lado, as afirmações diagnósticas qualificam a "integridade da pele" com a associação dos termos "prejudicada" e "risco para". A publicação de 2001 da classificação da NANDA traz detalhes que auxiliam a compreender melhor a proposta atual da NANDA.

Frente à classificação segundo Padrões de Respostas Humanas, a Taxonomia II apresenta termos cujos significados nos são mais familiares - fazem parte da enfermagem tradicional e contemporânea, e talvez sejam mais efetivos para a comunicação com outros profissionais, com o próprio cliente e com instâncias de planejamento dos serviços de saúde.

É importante que os enfermeiros não confundam a estrutura da Taxonomia II da NANDA, com a proposta dos Padrões Funcionais de Saúde ${ }^{(11-12)}$. A NANDA, como acima descrito, usou essa proposta para estruturar os diagnósticos em classes e domínios e é só agora, em 2001, que a publica. Antes disso, no entanto, provavelmente pela pouca familiaridade com os Padrões de Respostas Humanas (da antiga classificação da NANDA), os Padrões Funcionais de Saúde ${ }^{(11-12)}$ já estavam sendo utilizados em livros textos e em serviços nos Estados Unidos, tanto para estruturar a coleta dos dados, quanto para classificar os diagnósticos de enfermagem.

Especialistas em informática e gerentes de banco de dados são usuários primários da atual estrutura taxonômica. As enfermeiras clínicas poderão beneficiarse de conhecimentos sobre essa estrutura quando necessitarem recuperar informações rapidamente. Além disso, a idéia geral da estrutura taxonômica ajuda a enfermeira a compreender os diagnósticos que utiliza em sua prática clínica ${ }^{(6)}$.

A Taxonomia II é multiaxial e, por isso, é mais flexível que a Taxonomia I (monoaxial), facilitando a inclusão de novos diagnósticos. Um eixo, para o propósito da taxonomia da NANDA, é operacionalmente definido como uma dimensão da resposta humana considerada no processo diagnóstico. Os eixos que compõem a Taxonomia II são sete, com as seguintes definições.

Conceito diagnóstico (eixo 1) é definido como o principal elemento, ou parte essencial, a origem, o diagnóstico. Consiste de um ou mais substantivos como, por exemplo, integridade da pele. Tempo (eixo 2) é definido como a duração de um período ou intervalo. Os valores do eixo 2 são: agudo (< 6 meses), crônico (> 6 meses), intermitente (pára e inicia-se em intervalos, ciclos ou períodos), ou contínuo (sem interrupção). Unidade de cuidado (eixo 3) é definida como a população específica para a qual um diagnóstico de enfermagem é determinado (indivíduo, família, grupo e comunidade). Idade (eixo 4) é a extensão de tempo ou intervalo durante o qual o indivíduo tenha existido (feto, neonato, lactente, criança, criança em idade pré-escolar, criança em idade escolar, adolescente, adulto jovem, adulto de meia-idade, adulto idoso-jovem, adulto em meia-velhice, adulto idoso). Estado de saúde (eixo 5) designa a posição do indivíduo no "continuum" de saúde. Seus valores são: bem estar, risco, real. Descritor (eixo 6) indica o julgamento que limita ou especifica o significado de um diagnóstico de enfermagem (por exemplo: impaired = prejudicado). Topologia (eixo 7) designa partes ou regiões do corpo e suas estruturas anatômicas. Seus valores são, por exemplo: auditivo, intestinal, cardiopulmonar, cerebral, gastrintestinal, gustativo(a), intracraniano(a), urinário(a), membranas mucosas, oral, olfativo(a), neurovascular periférico(a), vascular periférico(a), renal, cutâneo(a), tátil, visual.

Esses eixos servem, então, para compor os enunciados diagnósticos nas situações clínicas. Considerando o enunciado "integridade da pele prejudicada" crônica, foram utilizados os eixos 1, 2 e 6 . 
Não é necessário e nem sempre adequado que se utilizem os sete eixos para enunciar um diagnóstico. É fácil imaginar que os sete eixos permitem inúmeras possibilidades de diagnósticos, aumentando a cobertura das situações clínicas pela Taxonomia.

A Taxonomia II permite uma linguagem mais rica para os diagnósticos de enfermagem porque possibilita múltiplas combinações dos termos entre os eixos e maior adaptação às atuais convenções de manuseio de terminologia entre bases de dados relacionadas que utilizam classificações - NANDA ${ }^{(6)}$, Nursing Interventions Classification (NIC) $)^{(13)}$ e Nursing Outcomes Classification $(\mathrm{NOC})^{(9-10)}$

Conforme a NANDA ${ }^{(6)}$, o cuidado de saúde mundial, onde os sistemas de saúde estão inseridos, deve ultrapassar fronteiras, limites de espaço, tempo e tecnologia; justificando tal classificação por razões de padronizar a nomenclatura dos diagnósticos de enfermagem; expandir o conhecimento de enfermagem

\section{REFERÊNCIAS BIBLIOGRÁFICAS}

1. Cruz DALM. A inserção do diagnóstico de enfermagem no processo assistencial. In: Cianciarullo TI, Gualda DMR, Melleiro MM, Anabuki MH, organizadoras. Sistema de assistência de enfermagem: evolução e tendências. São Paulo: Ícone; 2001.

2. Gordon M, Sweeney MA. Methodological problems and issues in identifying and standardizing nursing diagnosis. Adv Nurs Sci 1979; 2(1):1-15.

3. Braga CG. A resposta psicossocial de impotência e o "locus de controle" de pacientes no pós-operatório de cirurgia cardíaca [dissertação]. São Paulo (SP): Escola de Enfermagem/USP; 1999.

4. Silva AGI. Ensinando e cuidando com o processo diagnóstico em enfermagem. Belém (PA): Smith Produções Gráficas; 2001.

5. North American Nursing Diagnosis Association. Nursing Diagnoses: definitions and classification: 1999/2000. Philadelphia: NANDA; 1999.

6. North American Nursing Diagnosis Association. Nursing Diagnoses: definitions and classification: 2001/2002. Philadelphia: NANDA; 2001.

7. Cruz DALM. Fenômenos e intervenções de enfermagem: desatando nós conceituais. In: Garcia TR, Nóbrega MML, organizadoras. Sistema de Classificação da Prática de Enfermagem. João Pessoa (PB): ABEn; 2000.

8. Jenny J. Advancing the science of nursing with nursing diagnosis. Proceedings of the $11^{\text {th }}$ Conference; 1994; Glendale, CA: CINAHL.

9. Johnson M, Maas M. Nursing Outcomes Classification (NOC). St. Louis: Mosby; 1997. mediante a vinculação entre os diagnósticos, intervenções e resultados de enfermagem; determinar a relação custoeficácia, eficiência e qualidade do cuidado em enfermagem.

\section{CONSIDERAÇÕES FINAIS}

Este artigo contribui para atualizar os conhecimentos sobre a classificação de diagnósticos da NANDA, facilitando à enfermagem brasileira acompanhar a evolução do assunto. Estudos que explorem a estrutura da Taxonomia II contribuirão para melhor traçarmos os "mapas de território" do cuidar, com vistas à promoção da qualidade da assistência de enfermagem. "Não será fácil o trabalho, mas devemos continuar a construir um "mapa" que represente, cada vez melhor, a nossa prática e que sirva de instrumento para reconhecê-la e para colocá-la sob observação de nossos pares e da própria comunidade"(7).

10. Jonhson M, Maas M, Moorhead S. Nursing Outcomes Classification (NOC). $2^{\text {nd }}$ ed. St. Louis: Mosby, 2000.

11. Gordon M. Nursing diagnosis: process and application. New York: McGraw-Hill Book Company; 1982.

12. Gordon M. Nursing diagnosis: process and application. $3^{\text {th }}$ ed. New York: McGraw-Hill Book; 1994.

13. McCloskey JC, Bulechek GM. Nursing Interventions Classification (NIC). $3^{\text {rd }}$ ed. St. Louis: Mosby; 2000. 